\title{
A Comprehensive List of Undergraduate Bioinformatics Programs in the United States
}

\author{
Author: Hsi Chang \\ Indiana University-Purdue University Indianapolis
}

Abstract: A most comprehensive list of undergraduate bioinformatics programs in the United States. It includes 57 universities that offer bioinformatics majors. It is the most comprehensive collection in 2021, and it will help researchers on life science to foster further collaboration on bioinformatics education. Further, the list will help more American youths to pursue dreams in bioinformatics once the list is later published on Wikipedia.

Here, we present a comprehensive list of undergraduate bioinformatics programs in the United States.

The reason I do this research is when I was looking for undergraduate schools offering bioinformatics programs which I might attend, I couldn't find a full list and spent a lot of time doing the research. Even though some places have a collection, but they are outdated or have issues with verification: Carnegie Mellon University this year just updated its list to an interactive page but only has 31 programs on it including some programs no longer exist (Carnegie Mellon University School of Computer Science, 2021); University of North Carolina at Chapel Hill has a page that has not been updated since 2006 (University of North Carolina at Chapel Hill, 2006); some websites might also have a list but not accurately and been verified (Biology-Colleges.com, 2018); National Center for Education Statistics has interactive searching system which lists 71 bioinformatics programs without verification and lack of some schools including my institution IUPUI (National Center for Education Statistics, 2021). I think my time in the school searching process can contribute to a paper and additionally help others.

I first put keywords "bioinformatics undergraduate", "computational biology undergraduate", and combined with the state name in google, looking through all of the results; each search result will then be verified. Then do additional searching. I verified those schools listed on the mentioned websites. With the simple method, I have this comprehensive list.

It includes a total of 57 universities that offer the bioinformatics major.

To be clear, this list only covers universities that offer an independent major specifically to bioinformatics. The major name can be "bioinformatics", "computational biology", "biomedical informatics", "biomathematics", or "system biology". For the reason of standardization, I do not count the major considered "bioinformatics" as a concentration/track to a major in biology, computer science, informatics, etc., like University of Missouri-Kansas City (University of Missouri Kansas City, 2020). 
I believe this is the most comprehensive collection in 2021, and it will help researchers on life science to foster further collaboration on bioinformatics education. Further, the list will help more American youths to pursue dreams in bioinformatics once the list is later published on Wikipedia.

New England (Connecticut, Maine, Massachusetts, New Hampshire, Rhode Island, and Vermont)

$\underline{\text { Connecticut }}$

University of Saint Joseph

Bioinformatics, B.S.

https://www.usj.edu/academics/sasbe/biology/bioinformatics-bs/

Maine

Colby College

Computational Biology, BS

http://www.colby.edu/catalogue/requirements/bi/

Massachusetts

Wheaton College

Bioinformatics. BS

https://wheatoncollege.edu/academics/majors-minors/bioinformatics/

Worcester Polytechnic Institute

Bioinformatics \& Computational Biology, BS

https://www.wpi.edu/academics/departments/bioinformatics-computational-biology

New Hampshire

None

Rhode Island

Brown University

Computational Biology, ScB \& AB

https://www.brown.edu/academics/biology/undergraduate-education/undergraduate/computational-biology

$\underline{\text { Vermont }}$

None 
Mid-Atlantic (New Jersey, New York, and Pennsylvania)

New Jersey

Rowan University

Bioinformatics, BS

https://csm.rowan.edu/departments/mcb/academic-programs/undergraduate/bioinfo-bs.html

Ramapo College

Bioinformatics, BS

https://bioinformatics.ramapo.edu/bsbinf/index.html

Rutgers University-Camden

Computational and Integrative Biology, BS

https://ccib.camden.rutgers.edu/programs/undergraduate/

New York

University at Buffalo

Bioinformatics and Computational Biology, BS

https://arts-sciences.buffalo.edu/biological-sciences/undergraduate/programs/bioinformatics.html

Cornell University

Biometry and Statistics, BS

https://cals.cornell.edu/education/degrees-programs/biometry-statistics-major-and-minor

Rochester Institute of Technology

Bioinformatics and Computational Biology, BS

https://www.rit.edu/study/bioinformatics-and-computational-biology-bs

New York City College of Technology

Biomedical Informatics - BS

https://www.citytech.cuny.edu/biological/biomedical-informatics-bs.aspx

St. Bonaventure University

Bioinformatics, BS

https://www.sbu.edu/academics/bioinformatics

Pennsylvania

University of Pennsylvania

Computational Biology, BAS, BA

https://www.cis.upenn.edu/undergraduate/program-offerings/computational-biology/

https://catalog.upenn.edu/undergraduate/programs/biology-computational-biology-ba/

University of Pittsburgh

Computational Biology, BS

https://www.biology.pitt.edu/computational-biology 
Carnegie Mellon University

Computational Biology,BS

http://cbd.cmu.edu/education/undergraduate/index.html

East North Central (llinois, Indiana, Michigan, Ohio, and Wisconsin)

$\underline{\text { Illinois }}$

Loyola University Chicago

Bioinformatics, BS

https://www.luc.edu/bioinformatics/bsinbioinformatics/

Illinois Institute of Technology

Bioinformatics, BS

http://bulletin.iit.edu/undergraduate/colleges/science/biology/bs-bioinformatics/

$\underline{\text { Indiana }}$

Indiana University-Purdue University Indianapolis

Biomedical Informatics, BS

https://soic.iupui.edu/undergraduate/degrees/biomedical-informatics/

Rose-Hulman Institute of Technology

Biomathematics, BS

https://www.rose-hulman.edu/academics/academic-departments/mathematics/majors-minors.html\#Biomath

Michigan

Michigan Technological University

Bioinformatics, BS

https://www.mtu.edu/biological/undergraduate/bioinformatics/

$\underline{\text { Ohio }}$

Walsh University

Bioinformatics, BS

https://www.walsh.edu/bioinformatics-degree.html

Case Western Reserve University

Systems Biology, BS

https://biology.case.edu/undergraduate/bachelor-of-science-systems-biology/ 
$\underline{\text { Wisconsin }}$

University of Wisconsin-Parkside

Molecular Biology and Bioinformatics, BS

https://www.uwp.edu/learn/programs/molecularbiomajor.cfm

Marquette University

Bioinformatics, BS

https://www.marquette.edu/explore/major-bioinformatics.php

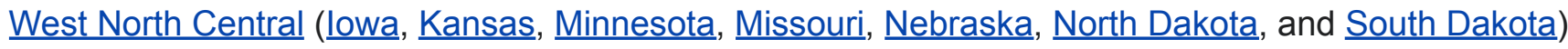

lowa

lowa State University

Bioinformatics and Computational Biology, BS

https://catalog.iastate.edu/collegeofliberalartsandsciences/bioinformaticsandcomputationalbiology undergraduat e/index.html

$\underline{\text { Kansas }}$

None

Minnesota

None

Missouri

University of Central Missouri

Bioinformatics BS

https://www.ucmo.edu/academics/programs/majors/bioinformatics-bs-43-653-biological-science-option/index.ph $\underline{\mathrm{p}}$

Nebraska

University of Nebraska Omaha,

Bioinformatics, BS

https://www.unomaha.edu/college-of-information-science-and-technology/school-of-interdisciplinary-informatics/ bioinformatics/bs-bioi.php

North Dakota

Minot State University

Bioinformatics, BS

https://www.minotstateu.edu/enroll/programs/bioinformatics.shtml 
$\underline{\text { South Dakota }}$

None

South Atlantic (Delaware, Florida, Georgia, Maryland, North Carolina, South Carolina, Virginia, District of Columbia, and West Virginia)

$\underline{\text { Delaware }}$

None

Florida

Florida State University

Computational Biology, BS

https://www.bio.fsu.edu/compbio/

Florida Institute of Technology.

Biomathematics, B.S.

https://www.fit.edu/programs/biomathematics-bs/

$\underline{\text { Georgia }}$

None

Maryland

University of Maryland, Baltimore County

Bioinformatics and Computational Biology, BS

https://biology.umbc.edu/undergrad/undergraduate-programs/binf/

Bowie State University

Bioinformatics, BS

https://bowiestate.edu/academics/colleges/college-of-arts-and-sciences/departments/natural-sciences/undergra duate-programs/bioinformatics-major/index.php

Towson University

Molecular Biology, Biochemistry \& Bioinformatics, BS

https://catalog.towson.edu/undergraduate/fisher-science-mathematics/molecular-biology-biochemistry-bioinform

atics/molecular-biology-biochemistry-bioinformatics/

North Carolina

None 
South Carolina

Presbyterian College

Computational Biology, BS

https://www.presby.edu/academics/undergraduate/academic-departments-programs/biology-department/require $\underline{\text { ments/ }}$

Claflin University

Bioinformatics, BS

https://www.claflin.edu/academics-research/schools-departments/school-of-natural-sciences-and-mathematics/d epartment-of-biology/bioinformatics

Virginia

Virginia Commonwealth University

Bioinformatics, BS

http://bulletin.vcu.edu/undergraduate/vcu-life-sciences/center-study-biological-complexity/bioinformatics-bs-conc entration-computational/

Virginia Polytechnic Institute and State University.

Systems Biology, BS

https://www.registrar.vt.edu/graduation-multi-brief/checksheets.html

Washington

Whitworth University

Bioinformatics, B.S.

https://www.whitworth.edu/cms/academics/undergraduate-majors-and-programs/bioinformatics-bs/

District of Columbia

None

West Virginia

None

East South Central (Alabama, Kentucky, Mississippi, and Tennessee)

Alabama

University of Alabama Birmingham

Bioinformatics, BS

https://www.uab.edu/cas/bioinformatics/

Kentucky

None 
Mississippi

None

$\underline{\text { Tennessee }}$

Lipscomb University

Computational Biology, BS

https://www.lipscomb.edu/academics/programs/computational-biology

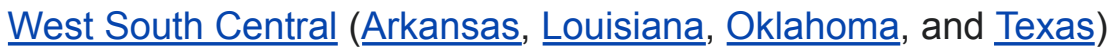

Arkansas

None

Louisiana

None

Oklahoma

None

Texas

St. Edward's University

Bioinformatics, BS

https://www.stedwards.edu/undergraduate/bioinformatics

St. Mary's University

Bioinformatics, BS

https://catalog.stmarytx.edu/undergraduate/majors-programs/science-engineering-technology/bioinformatics/\#tr ackstext

Baylor University.

Bioinformatics, Bachelor of Science in Informatics

https://www.ecs.baylor.edu/index.php?id=961235

Our Lady of the Lake University

Biomathematics, BS

https://www.ollusa.edu/cas/programs/mathematics-and-biomathematics/index.html 
Mountain (Arizona, $\underline{\text { Colorado, Idaho, Montana, }}$ Nevada, New Mexico, Utah, and Wyoming)

Arizona

University of Arizona

Bioinformatics, BS

https://eeb.arizona.edu/undergraduate/majors-minors/bs-bioinformatics

Arizona State University

Biomedical Informatics, BS

https://chs.asu.edu/programs/biomedical-informatics

$\underline{\text { Colorado }}$

None

Idaho

Lewis-Clark State College

Bioinformatics BA/BS

http://catalog.Icsc.edu/academic-programs/natural-sciences-mathematics-division/baccalaureate-degrees/bioinf ormatics-ba-bs/

Montana

None

Nevada

None

$\underline{\text { Nevada }}$

None

New Mexico

None

$\underline{\text { Utah }}$

Brigham Young University

Bioinformatics, BS

https://biology.byu.edu/bioinformatics

Dixie State University

Bioinformatics, BS

https://catalog.dixie.edu/programs/biology/bioinformatics-bs/ 
Wyoming

None

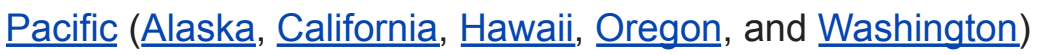

$\underline{\text { Alaska }}$

None

California

California State University-San Bernardino

Bioinformatics, BS

https://www.csusb.edu/cse/programs/bs-bioinformatics

University of California, San Diego

Bioinformatics, BS

https://bioinformatics.ucsd.edu/node/8

University of California, Santa Cruz

Biomolecular Engineering and Bioinformatics, BS

https://admissions.sa.ucsc.edu/majors/beb

Harvey Mudd College

Mathematical and Computational Biology, BS

https://www.hmc.edu/mathematics/program/majors/mathematical-computational-biology-major/

$\underline{\text { Hawaii }}$

None

Oregon

Pacific University

Bioinformatics, BS

https://www.pacificu.edu/bioinformatics

Oregon State University

Biological Data Sciences, BS

https://catalog.oregonstate.edu/college-departments/agricultural-sciences/botany-plant-pathology/biological-dat a-sciences-bs-hbs/ 
Washington

Whitworth University

Bioinformatics, B.S.

https://www.whitworth.edu/cms/academics/undergraduate-majors-and-programs/bioinformatics-bs/ 


\section{Citation}

Biology-Colleges.com. (2018). Biology schools offering biomathematics and bioinformatics bachelor's degrees.

https://biology-colleges.com/bioinformatics-and-biometrics/bachelor

Carnegie Mellon University School of Computer Science. (2021). Undergraduate

Computational Biology/Bioinformatics Programs.

http://careers.cbd.cmu.edu/listing-category/ugrad-compbio-bioinformatics/

National Center for Education Statistics. (2021). College navigator - Search results.

https://nces.ed.gov/collegenavigator/?s=all\&p=26.1103+26.1199+26.1104\&l=93\&of=2

$\&$ od $=0 \& p g=1$

University of North Carolina at Chapel Hill. (2006). Bioinformatics programs in the United States.

https://ils.unc.edu/informatics_programs/doc/Bioinformatics_2006

University of Missouri Kansas City. (2020). Bachelor of Science: Biology - Bioinformatics Emphasis

https://catalog.umkc.edu/colleges-schools/sbc/biology/bachelor-of-science-bioinformati cs/ 